\title{
Context Aware Intelligent Wallet for Healthcare
}

\author{
Arif M. Bhatti, Mehedi Masud \\ College of Computers and Information Technology \\ Taif University \\ Taif, KSA
}

\begin{abstract}
Use of technology in healthcare to improve quality of life, real-time patient monitoring and large scale studies of human behavior is very active area of research. Advances in ubiquitous communication, pervasive computing and ambient intelligence has given rise to smart objects that can communicate and cooperate to build network of things to perform physical computing. Pervasive healthcare system use smart objects to collect data about physical, physiological and behavioral processes. Goal of this research is to design an intelligent wallet for individuals that collects and stores human bio-signals using medical sensors, and context data using environment and motions sensors on personal smart devices. The wallet detects abnormal pattern and shares data and findings with appropriate persons or medical systems. This paper presents system architecture and proof-of-concept implementation of a context aware intelligent wallet for healthcare. Core of the system is designed around user and personal smart devices.
\end{abstract}

\section{General Terms}

Pervasive Computing, Healthcare, Bio-signal Sensors, Context Aware

\section{Keywords}

Personal healthcare, smart environment, medical sensors, context sensors

\section{INTRODUCTION}

Advances in personal computing, ubiquitous communication technologies, web protocols, and sensor hardware made it possible to create smart environments for social as well as personal well being managed and controlled by the users. This is evident from a huge number of apps on android and IOS app stores.

To store health related information, early healthcare information systems used proprietary protocols to store and retrieve patient data that resulted in inter-operability problem among different system deployed by different organizations. To address the problem of interoperability several electronic health records (EHR) standards [1] are under development. EHR includes information such as observations, laboratory tests, diagnostic image reports, treatments, therapies, drugs administered, patient identification, allergies and legal permissions.

EHR standards are used by the hospitals and doctors, while personal health record (PHR) empowers the patient to manage personal medical records, vital sign reading, prescription, lab test and other health related information [3, 4]. A patient can modify and update information in PHR and decides who can have the access to what part of the data.

Interoperability problem among different systems can be solved by using service oriented architecture (SOA) and web services. SOA based systems expose external interfaces as web services while hiding internal implementation details and standards used [2]. Systems designed for EHR, PHR and personal health care can communicate and share information as long as they provide web services interface.

Ubiquitous communication technologies such as Zigbee, Bluetooth, GSM, GPRS, WiFi, WiFi Direct, NFC and 4G have enabled computing objects to communicate with other objects. Communication ability of objects has created a new kind of Internet called The Internet of Things (IoT) [5] that has enabled communication pattern of anytime, anywhere, anyone and anything.

Physical computing is about recognition of changes by objects in a physical environment; objects actively interact with each other to perform some meaningful actions. Ambient intelligence is the ability to infer meaningful information from communication among objects. Researchers are using IoT and ambient intelligence in healthcare to monitor elderly patient to provide medical assistance and to improve quality of life [5].

Healthcare systems based on smart objects, IoT and ambient intelligence are data intensive. Even though smart environments generate a huge amount of data, not all data is needed for future use. Preprocessing of this data is required to aggregate it into meaningful information for long term storage. Security, privacy and trust are the most important part of any healthcare information systems [4].

In a user-centric system, smart objects communicate with user's personal devices such as mobile phone or tablet. User's devices are the main conduit between the smart environment and an information system that can store and process data.

In an intelligent system based on smart objects, context is very important to interpret the data and take some intelligent actions. A medical sensor reading may be abnormal if a user is in a sitting position but same reading is okay if the user is running. A reading of body temperature in an air conditioned room should have a different meaning than a reading under the sun. Context Aware computing [14] is about collection of implicit context and use of this context for intelligent decision making. Decision supports systems are needed to take actions based on the data in case of emergency or some abnormal and life threatening pattern in sensor reading.

This paper focuses on creation of smart healthcare environment for individuals, where personal information, biosignal from medical sensors, and context in which bio-signals were captured are regularly updated in a user-centric wallet maintained in the cloud. Intelligence component of the wallet processes its contents for pattern of interest to detect abnormal health behavior.

\section{SYSTEM ARCHITECTURE}

Goal of this research is to create an intelligent wallet for individuals to store their bio-signals and context in which biosignals were collected. In case of abnormal pattern in sensor 
reading, the wallet intelligently shares data and findings with appropriate persons or medical systems. Information in the wallet is managed and controlled by the user-defined policies with full control on how this data will be used.

System is designed in a layered architecture as shown in figure [1]. First layer collects and processes data on sensor nodes in the smart healthcare environment. Special hardware is needed to implement sensor nodes. Second layer collects formatted data from multiple sensor nodes and context sensors. After filtering and aggregation this information is sent to the intelligent wallet in upper layer. Functionality in this layer is designed to be implemented on personal devices such as smart phones and tablets.
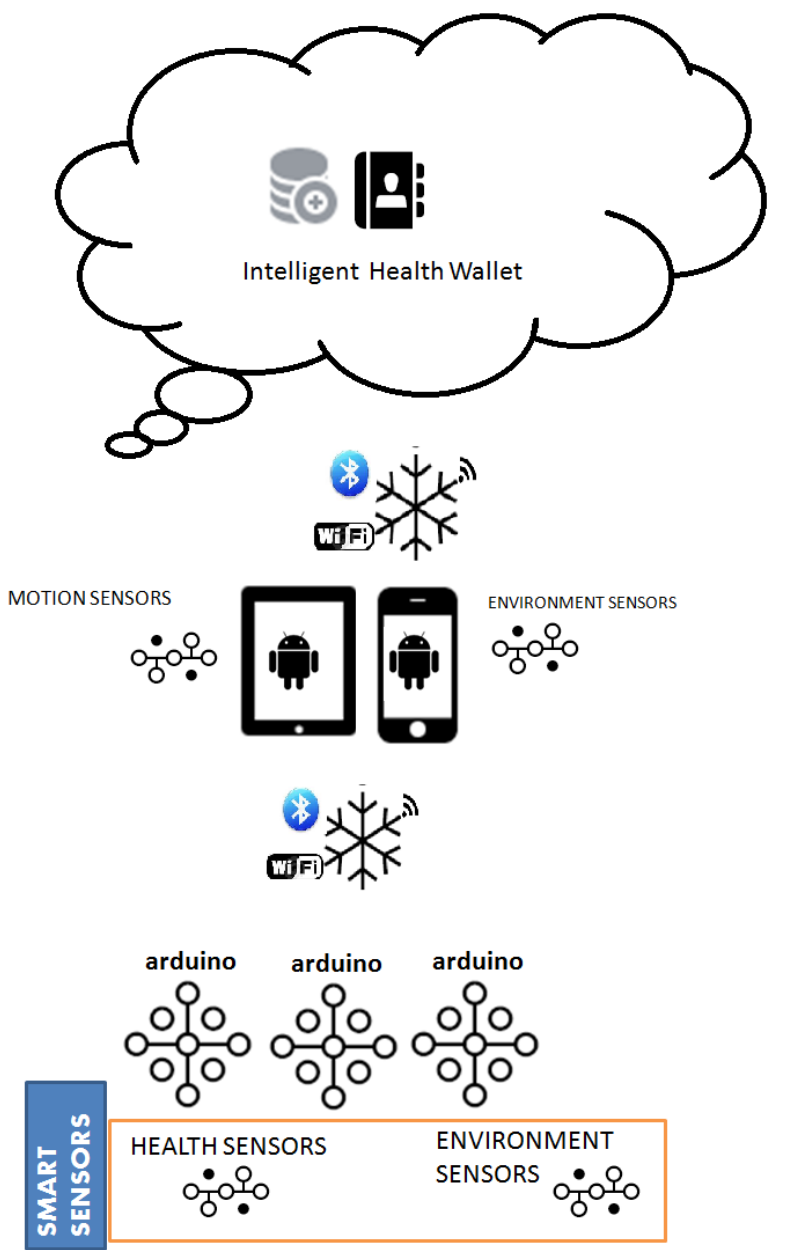

Fig 1: System architecture of intelligent wallet for healthcare. Main component of the architecture are: Sensor nodes, personal gateways to collect data from sensor node and send this data to the intelligent wallet on server or in the cloud. Wallet has intelligence component to detect abnormal patterns.

Third layer receives data from personal devices and stores it in the database. An intelligence engine performs feature extraction needed to perform intelligent operations on stored data. After detection of an abnormal or suspicious pattern in wallet data, a message is sent to appropriate persons or systems based on user preferences.

Rest of this section discusses the role of medical and context sensors, data collection and processing on sensor nodes, functionality in personal gateway devices, and data storage in the healthcare wallet.

\subsection{Bio-Signal and Context Sensors}

Focus of this research is user-centric and user-controlled monitoring of individuals in the following categories.

1. Personal Care

2. Physical Fitness

3. Remote Self-managed Healthcare

4. Patient in ICU for post-op care

5. Patient under medical observation

Possible medical and context sensors for the system are discussed in rest of the section.

\subsubsection{Bio-Signal Sensors}

Medical practitioners use bio-medical sensors to detect abnormalities in human body functioning. Reading from multiple sensors is required to diagnose the cause of patient's visit. Following is a list of medical sensors that can be used in a smart environment for healthcare.

- Pulse and Oxygen Sensor provides pulse rate and oxygen level in the blood.

- Air Flow Sensor detects breathing out activity and its intensity that can identify abnormal breathing patterns and possible diseases that may have caused the breathing pattern $[6,7]$.

- Body Temperature Sensor measures human body temperature. It is of great medical importance as a number of diseases are accompanied by characteristic changes in body temperature. Changes in body temperature reflect state of internal body parts.

- Electrocardiogram (ECG) Sensor is a diagnostic tool that is routinely used to assess the electrical and muscular functions of the heart. Periodic waveform generated by this sensor has different components, and variation in the components identifies the type and seriousness of heart abnormality [8,9].

- Glucometer Sensor is used to detect blood sugar level and used very frequently and regularly by diabetics patients to manage their sugar level.

- Galvanic Skin Response (GSR) Sensor is used to detect changes in sweat level, which is reflective of changes in emotions or anxiety level.

- Blood Pressure Sensor measure the pressure of blood in the arteries as it is pumped around the body by the heart. Blood pressure is recorded as two numbers, the systolic pressure and the diastolic pressure.

- Electromyogram (EMG) Sensor measures the electrical activity of muscles at rest and during contraction. EMG is used as a diagnostics tool for identifying neuromuscular diseases, assessing lowback pain, kinesiology, and disorders of motor control.

- Body Position Sensor is not really a bio-signal sensor. It is an accelerometer sensor to monitors different body positions: standing/sitting, supine, prone, left and right. Reading from this sensor along with Airflow sensor can help in identifying abnormalities in breathing patterns [6, 7].

\subsubsection{Context Sensor}

Medical facilities have controlled environments and bio-signal readings have definitive interpretation. Focus of our work is in 
un-controlled user-centric smart environment where it is not possible to use bio-signal information alone without knowing the environment where the bio-signals were collected. Following sensors are available on personal devices such as smart phones and tablets [11], which is at the center of our system.

- Motion Sensors: Accelerometer, Gyroscope, Gravity, Linear Acceleration and Orientation.

- Environment Sensor: ambient light, ambient temperature, pressure and elevation, magnetic field, humidity, and proximity.

- Location Sensor: GPS and network-based location

- Video Sensor: IP camera and smart phone cameras

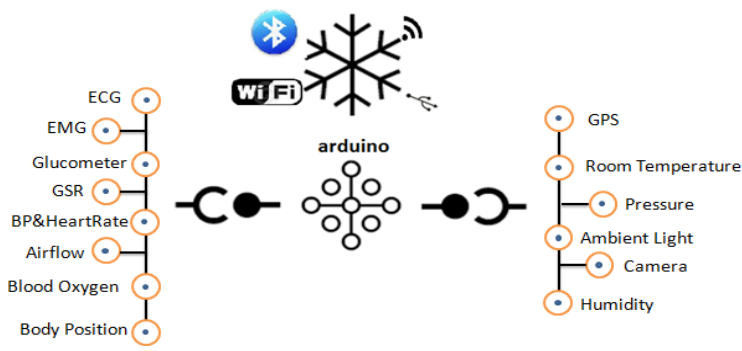

Fig 2: A sensor node based on Arduino microprocessor board with sensors that can be connected with the node. It may not be possible to connect all sensors simultaneously. Node is equipped with USB and wireless communication technologies.

\subsection{Smart Node and Data Filtering}

Smart environment for healthcare has medical sensors to detect vital signs of individuals as well as sensors to detect motion, environment, location and visual information needed to interpret bio-signals to detect health hazard or a health problem.

Smart node is a microprocessor based embedded system that can have any combination of medical as well as environment sensors attached with. Choice of sensors will depend on the user profile as listed in section 2.1. For a patient in ICU, Airflow, ECG, temperature, and oxygen level in blood is more important while for a person with personal care needs heart rate, location, temperature and humidity may be of interest.

Sensor node collects signals from attached sensors, and converts analog signals into digital before these signals can be used for further processing. Sequence and frequency of sensor reading is encoded in a program that runs on microprocessor board. For Arduino-based smart node it is possible to write customized program and upload it on the board. While sensor nodes based on other architecture may not have this facility to write and upload a customized program.

User's personal devices also act as smart nodes as most of the smart phones have environment sensors, motion sensors and possibly some bio-signal sensors [11]. Since smart phone are general purpose computing devices, this mean a customized program can be installed on these devices to define sequence and frequency of data collection.

Personal gateways are user devices to collect data from sensor nodes in the user's smart environment. Connectivity between personal gateway devices and sensor nodes can be established through USB cable, Bluetooth pairing, WI-Fi Direct, Wi-Fi or Zigbee networking technologies.
A sensor node collects data from all attached sensors and sends it to the personal gateway. Program running on the sensor node should know the following.

- Order of sensors for data collection

- Number of readings from each sensor

- Time-interval between successive readings from the same sensor

- Does all sensor need to be read on each iteration or in every iteration different subset of sensor should be read?

- How often each sensor should be read?

- What will be the format of data sent to the personal gateway?

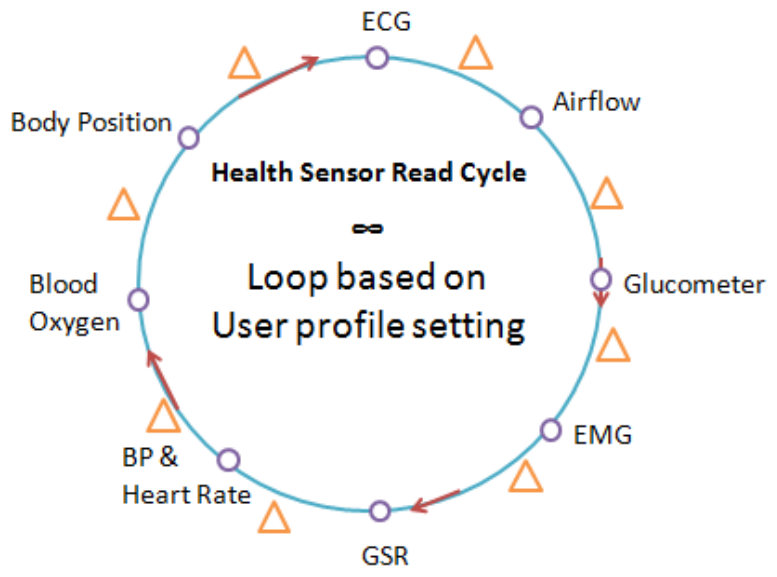

$\triangle=20 \mathrm{~ms}$ time lag

Fig 3: A round robin approach to collect data from each sensor based on the user profile. Different user profile may have different subset of sensors. In Arduino-based sensor node, a program can be written in a subset of

$\mathrm{C} / \mathrm{C}++$ to implement sensor ordering, frequency of reading and data transmission format.

\subsection{Personal Gateways}

Smart devices are ubiquitous in the form of smart phones, tablets, smart watches, eye glasses and other form of wearable devices. These devices are smart in nature as these devices have multi-core processors for computing, a wide range of networking technologies to interact with network-enabled devices, a wide range of sensors capable of collecting motion and environment information, and ability to communicate with cloud-based services using Internet protocols.

Personal devices are designed to be used in interactive mode and these devices are not very friendly for long-running periodic data-processing applications. Writing a program to periodically collect sensor data require a significant effort to ensure that operating system of smart device does not kill you program.

Collection of data from a sensor node is a periodic activity. Frequency of this activity depends on the user profile. For a patient in ICU, sensor readings have to be continuous while for a health enthusiast data collection from sensors will be an infrequent activity. 


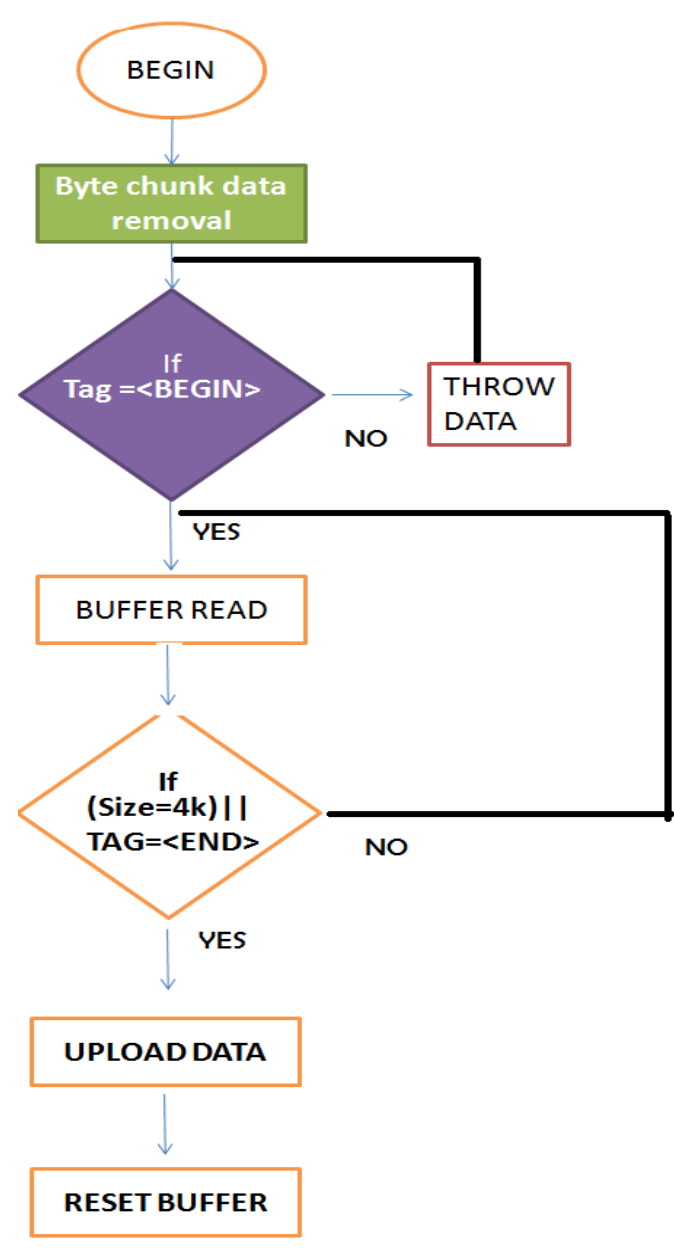

Fig 4: Algorithm to receive data from Arduino-based sensor node. To detect start of data, a $<$ BEGIN> tag has to be in a stream. Once start tag is found, data is collected in a buffer as soon as the $\langle$ END $>$ tag is found data from sensor node is processed and uploaded on the server in the cloud.

To reduce power consumption and mobile data network usage sensor node and personal gateway agree on data format and frequency of data to be read. In our proof-of-concept implementation, for each set of complete data, sensor node inserts $<$ BEGIN $>$ tag to mark the start of the data and <END> tag marks end of data. As soon as personal gateway starts reading data from the sensor node, it looks for $\langle$ BEGIN $\rangle$ tag. Until this tag is received, all received data is ignored.

Once the start tag is found, personal gateway starts buffering sensor data until it receives an $\langle\mathrm{END}\rangle \mathrm{tag}$. As soon as the end tag is received, further data from sensor node is ignored and buffered data is packaged and handed over to a background thread that sends this data to a web service. This service collects and verifies the data before inserting this data in user's healthcare wallet. Figure 4 shows a flowchart of algorithm implemented on android devices.

Android has its own event driven interface to get data from device sensors. It is not possible to get data from any sensor directly at will. To get data from any sensor on an android device, first the program has to register with sensor manager and register an event listener as call back function. Whenever there is an event from the sensor, android invokes the call back function and function extracts sensor data from the sensor event object. Same approach applies for GPS or networked-based location request from android OS.

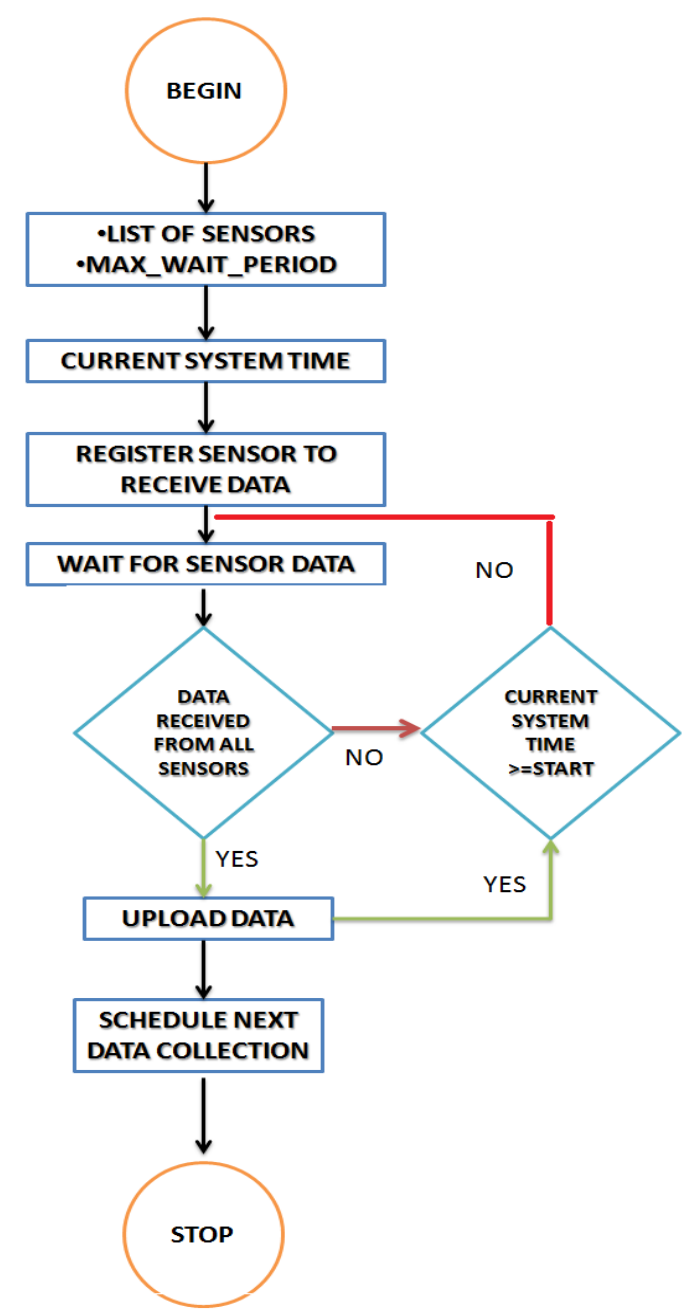

Fig 5: Algorithm to collect sensor data from an android device. It initializes the list of sensors to be read and initializes max time to wait for sensor readings to be available. If data from all sensors is available before the time-out or timer expires then available data is formatted and uploaded.

An algorithm to collect data from sensors on android-based personal gateways is shown in figure 5. Algorithm initializes the list of sensors to be read. To avoid infinite blocking, a timeout mechanism is introduced. First, sensors in the list are registered with the device OS. Algorithm keeps tracks of the sensor that have already been read. When all the sensors have been read or time-out happened before all sensor data received, collected sensor data is passed to a background thread that packages the data and uploads it in the user's wallet in the server.

\subsection{Context Aware Intelligence Wallet in the Cloud}

Wallet receives data from personal gateways; stores the received data in the database. Feature extraction is performed on data to prepare it to be used in intelligent decision making as shown in figure 6. Data in the wallet can be viewed by the user himself or anyone authorized by him.

Intelligence component uses knowledge base and user data in the wallet to find out any abnormal pattern. Once a pattern is detected, then this component initiates a protocol to find out 
who should be contacted? How severe is the abnormality? We categorize abnormality into following four categories:

- Caution: User is informed through his preferred mode of communication. He can view data and history for self analysis and further actions.

- Minor: User is advised about the abnormality and data is shared on his advice with his medical doctor. $\mathrm{He}$ can choose to ignore or share data with anyone he think should know about it.

- Major: In addition to user, his medical doctor and other authorized people are informed about the abnormality. How much data and with whom it should be shared depends on user profile

- Emergency: Personal medical doctor, immediate contact person and may be emergency services should be contacted. Abnormal data along with history is shared with anyone who is authorized.

\section{IMPLEMENTATION}

A proof-of-concept system was implemented that uses a subset of bio-signal and context sensors listed in section 2.1. Hardware needed for this research includes bio-signal sensors, motion sensors, environment sensors, location sensors, microprocessor boards and smart devices to collect and process sensor data. To implement sensor node, e-Health Sensor Platform V2.0 for Arduino kit [13] is used. Arduino is an open source programmable platform that provides hardware and software for research. We implemented data filtering and aggregation algorithms on Arduino UNO board. The board was connected with android device using USB host functionality.

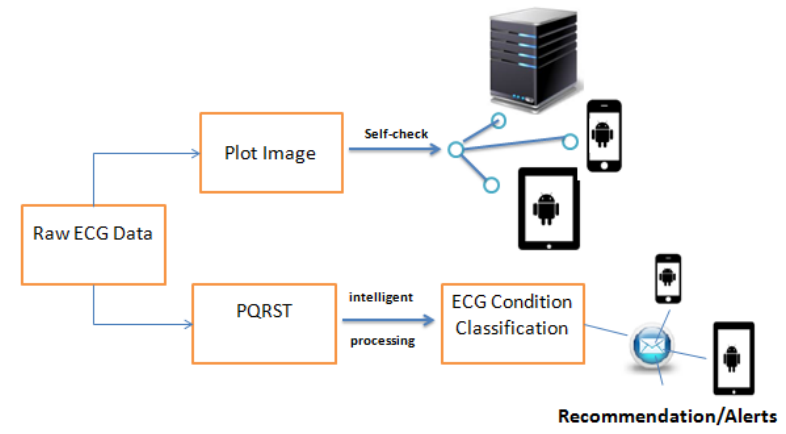

Fig 6: Once ECG data is received by the wallet three things happens: (1) data is stored as it is for future use, (2) A graph is generated to be shared and stored as image, and (3) features extraction algorithm process the data for

features to be used by the intelligence component to identify abnormal behavior.

Sensor node based on bitalino kit [12] has three bio-signal sensors, one environment sensor and one motion sensor. Unlike Arduino, bitalino board cannot be programmed. It allows configuration using some commands to control the frequency of data collection and the type of data to be collected.

Personal gateway functionality was implemented on androidbased devices: Google Nexus 7 tablet and Samsung Galaxy S4. These devices are equipped with cameras, GPS, environment and motion sensors, and networking technologies such as Wi-Fi, NFC, Bluetooth 4.0, GSM, HSPA+, and LTE.

\subsection{Health Wallet in the Cloud}

In proof-of-concept implementation, Wallet interface is a RESTful web service. Android-based devices are used to collects sensor data. Collected data is sent in JSON format to the wallet periodically. Wallet processes the incoming data and stores it in a MySQL database. Every storage request contains MAC address of the personal gateway.

Context data generated from environment, motion and location sensors is simple in nature, few well defined values for each reading. Environment sensor usually have single value, location sensor has two values longitude and latitude, and motion sensors have three values as $\mathrm{x} \mathrm{y}$ and $\mathrm{z}$. as shown in figure 7.

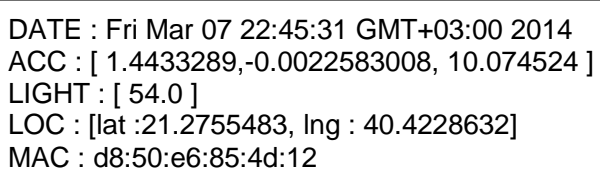

Fig 7: Data sent from personal gateway device (Nexus 7) and received at the server in the above format for ondevice sensors. Data starts with date as time stamp, accelerometer $x y$ and $z$ values, light sensor, location via GPS and MAC address of the device. This data is stored in the user wallet.

Data from bio-signal sensors such as Airflow, ECG, EMG and GSR is in waveform digitized from analog signals. Frequency of data point collection for bio-signals is an important issue for communication, power consumption and storage reasons. High frequency of data collection generates too much data that can overrun the available storage, and drains the device batteries. A lower frequency may have impact on data accuracy, as important information is lost especially in ECG. In proof-of-concept system, focus was only on Airflow and ECG bio-signals.

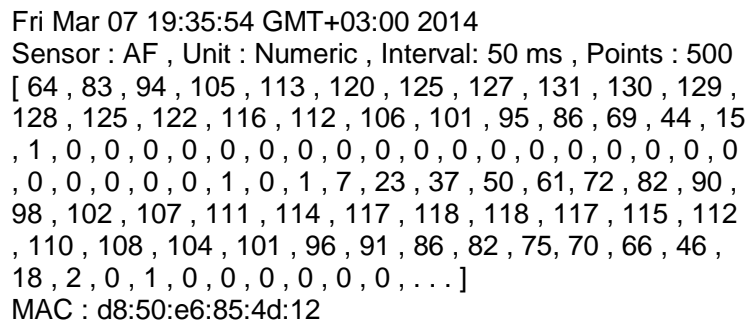

Fig 8: Airflow sensor data collected on sensor node processed on personal gateway and received by the wallet in this format. Data starts with timestamp, followed by sensor name, interval between each pair of data points and number of points in the data. Last value is the MAC address of personal gateway device that uploaded the data in the wallet.

Raw airflow data as shown in figure 8 is stored in the wallet. Intelligent component of the wallet requires features such as breathing rate per minutes and intensity of breathing to identify breathing abnormalities listed in $[6,7]$. Data collection for airflow sensor was performed at a lower frequency of $50 \mathrm{~ms}$ interval between any two data points. ECG waveform is very complex and has features such as Pwave, PR Interval, QRS complex, and RR interval [9]. Variation in these features identifies different heart problems. Complex nature of ECG waveform requires more frequent sensor readings to avoid missing any important information. 
Fri Mar 07 19:36:20 GMT+03:00 2014

Sensor : ECG , Unit : $\mathrm{mV}$, Interval: $10 \mathrm{~ms}$, Points: 500

[ $-0.085,-0.09,-0.06,-0.08,-0.05,-0.06,-0.045,-0.015$, -

$0.025,-0.005,-0.005,0.04,0.045,0.09,0.1,0.16,0.16$

$0.19,0.175,0.165,0.11,0.085,0.025,0.005,0.03,0.09$,

$0.07,0.11,0.095,0.12,0.115,0.125,0.105,0.135,0.125$,

$0.14, \ldots]$

MAC : d8:50:e6:85:4d:12

Figure 9, shows ECG data collected at sensor node with $10 \mathrm{~ms}$ interval between any two points.

Fig 9: ECG Sensor data collected at a sensor node, processed and uploaded in the wallet. Data start with a timestamp, followed by sensor name, data unit, interval between two points and total points in data message. Last value is MAC address of the personal gateway.
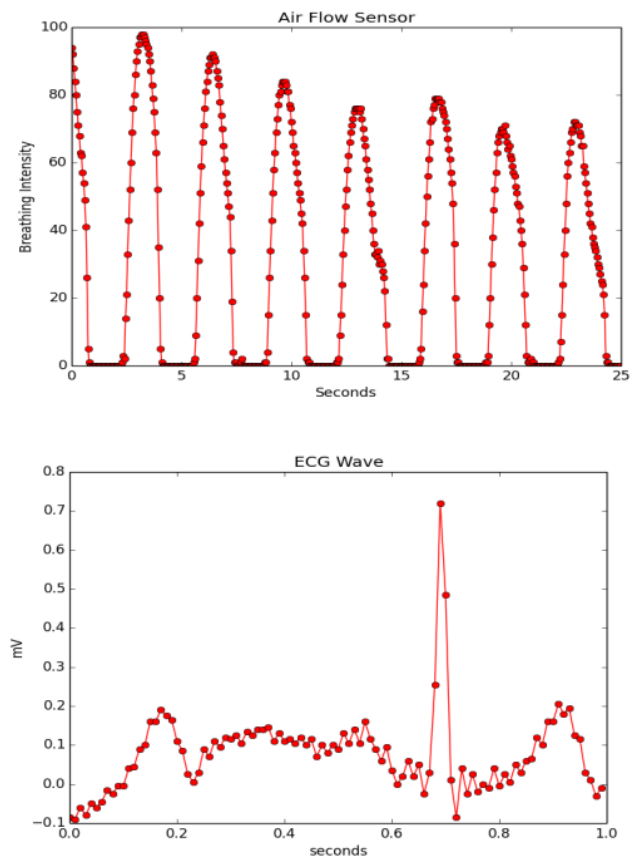

Fig 10: Airflow and ECG graphs generated for wallet data as shown in figures 8 and 9.

\section{CONCLUSION}

This paper presents architecture of a user-centric context aware intelligent wallet for personal health and medically supervised monitoring of individuals'. System design is based on layered approach. System functionality was implemented in sensor nodes, personal gateways, and cloud-based wallet with intelligence component. In proof-of-concept system, sensor node and personal gateways were implemented using Arduino UNO board and android-based devices. Restful web service and MySQL database were used to store data in the cloud. Intelligence component of the wallet was implemented using database triggers.

In future research, we are interested in simulating a very large number of intelligent wallets using bio-signal data from PhysioBank [10] to implement abnormality detection algorithms based on complex event processing (CEP) and data mining techniques.

\section{REFERENCES}

[1] Marco Eichelberg, Thomas Aden, Riesmeier, Asuman Dogac, and Gokce B. Laleci. 2005. A survey and analysis of Electronic Healthcare Record standards. ACM Comput. Surv. 37, 4 (December 2005)

[2] Jason H. Christensen. 2009. Using RESTful webservices and cloud computing to create next generation mobile applications. In Proceeding of the 24th ACM SIGPLAN conference companion on Object oriented programming systems languages and applications (OOPSLA '09). ACM, New York, NY, USA

[3] Ali Sunyaev, Dmitry Chornyi, Christian Mauro, Helmut Krcmar, "Evaluation Framework for Personal Health Records: Microsoft HealthVault Vs. Google Health," Hawaii International Conference on System Sciences, pp. 1-10, 2010 43rd Hawaii International Conference on System Sciences, 2010

[4] David Daglish, Norm Archer, "Electronic Personal Health Record Systems: A Brief Review of Privacy, Security, and Architectural Issues," Privacy, Security, Trust and the Management of e-Business, World Congress on Privacy, Security, Trust and the Management of e-Business, 2009

[5] A. Dohr, R. Modre-Opsrian, M. Drobics, D. Hayn, G. Schreier, "The Internet of Things for Ambient Assisted Living," Information Technology: New Generations, Third International Conference on, pp. 804-809, 2010 Seventh International Conference on Information Technology, 2010

[6] Breathing

Patterns; http://en.wikipedia.org/wiki/List_of_terms_of_lung_size _and_activity

[7] Abnormalities in rate and rhythm of breathing; http://quizlet.com/6961218/abnormalities-in-rate-andrhythm-of-breathing-flash-cards/

[8] ECG Rythms; http://www.ambulancetechnicianstudy.co.uk/rhythms.ht $\mathrm{ml}$

[9] ECG Basics; http://lifeinthefastlane.com/ecglibrary/basics/

[10] Goldberger AL, Amaral LAN, Glass L, Hausdorff JM, Ivanov PCh, Mark RG, Mietus JE, Moody GB, Peng CK, Stanley HE. PhysioBank, PhysioToolkit, and PhysioNet: Components of a New Research Resource for Complex Physiologic Signals. Circulation 101(23):e215e220 [Circulation Electronic Pages; http://circ.ahajournals.org/cgi/content/full/101/23/e215]; 2000.

[11] Android

Sensors; http://developer.android.com/guide/topics/sensors/

[12] Guerreiro, José, Raúl Martins, Hugo Silva, André Lourenço, and Ana Fred. "BITalino: A Multimodal Platform for Physiological Computing." In Proc. of the 10th ICINCO Conf. 2013

[13] e-Health Sensor Platform V2.0 for Arduino kit; http://www.cooking-hacks.com/ehealth-sensorscomplete-kit-biometric-medical-arduino-raspberry-pi

[14] Pilar Castro Garrido, Guillermo Matas Miraz, Irene Luque Ruiz, Miguel Angel Gomez-Nieto, "A Model for the Development of NFC Context-Awareness Applications on Internet of Things," Near Field Communication, International Workshop on, pp. 9-14, 2nd International Workshop on Near Field Communication, 2010. 